\title{
The method of lower and upper solutions to the Darboux problem for partial differential inclusions
}

\author{
Mouffak Benchohra and S. K. Ntouyas
}




\title{
THE METHOD OF LOWER AND UPPER SOLUTIONS TO THE DARBOUX PROBLEM FOR PARTIAL DIFFERENTIAL INCLUSIONS
}

\author{
M. BENCHOHRA AND S. K. NTOUYAS
}

[Received: February 28, 2003]

\begin{abstract}
Aвstract. The method of lower and upper solutions combined with a fixed point theorem for condensing multivalued maps due to Martelli is used to investigate the existence of solutions to an hyperbolic differential inclusion.
\end{abstract}

Mathematics Subject Classification: 35L70, 35L20, 35R70

Keywords: Hyperbolic differential inclusion, convex valued multivalued map, existence of upper and lower solutions, condensing map, fixed point

\section{INTRODUCTION}

This note deals with the existence of solutions to the following hyperbolic differential inclusion (Darboux problem):

$$
\begin{gathered}
\frac{\partial^{2} u(x, y)}{\partial x \partial y} \in F(x, y, u(x, y)), \quad(x, y) \in J \times J, \\
u(x, 0)=f(x), \quad u(0, y)=g(y)
\end{gathered}
$$

where $J=[0, T], F: J \times J \times \mathbb{R} \rightarrow 2^{\mathbb{R}}$ is a compact and convex valued multivalued map and $f, g: J \rightarrow \mathbb{R}$.

The method of upper and lower solutions has been successfully applied to study the existence of multiple solutions for initial and boundary value problems of the first and second order partial differential equations. This method has been used only in the context of single-valued partial differential equations. We refer to the books by Carl and Heikkilae [6], Heikkilae and Lakshmikantham [10], Ladde et al. [15], to the papers by Agarwal [1], Agarwal and Sheng [2], Blakley and Pandit [5], Lakshmikantham and Pandit [14], Pandit [18] and the references cited therein.

In this note we shall establish an existence result for the problem (1.1), (1.2). Our approach is based on the existence of upper and lower solutions and on a fixed point theorem for condensing multivalued maps due to Martelli [17]. Other existence results for the Darboux problem for partial differential equations and inclusions by 
the aid of other tools can be found in the recent monograph by Kamont [12] and in the papers $[4,7,8,13,19]$.

\section{Preliminaries}

In this section, we introduce notations, definitions, and preliminary facts from multivalued analysis which are used throughout this paper. In the sequel, we set $\mathcal{J}=J \times J$.

In what follows, $C(\mathcal{J}, \mathbb{R})$ is the Banach space of continuous functions from $\mathcal{J}$ into $\mathbb{R}$ with the norm $\|z\|_{\infty}:=\sup \{|z(x, y)|:(x, y) \in \mathcal{J}\}$ for each $z \in C(\mathcal{J}, \mathbb{R})$.

The condition

$$
u \leq \bar{u} \quad \text { if and only if } \quad u(x, y) \leq \bar{u}(x, y) \quad \text { for all } \quad(x, y) \in \mathcal{J}
$$

defines a partial ordering in $C(\mathcal{J}, \mathbb{R})$. If $\underline{u}, \bar{u} \in C(\mathcal{J}, \mathbb{R})$ and $\underline{u} \leq \bar{u}$, we put

$$
[\underline{u}, \bar{u}]=\{u \in C(\mathcal{J}, \mathbb{R}): \underline{u} \leq u \leq \bar{u}\} .
$$

Let $(X,|\cdot|)$ be a Banach space. A multivalued map $G: X \rightarrow 2^{X}$ is convex (closed) valued if $G(x)$ is convex (closed) for all $x \in X$. $G$ is bounded on bounded sets if $G(B)=\bigcup_{x \in B} G(x)$ is bounded in $X$ for any bounded set $B$ of $X$ (i.e. $\sup _{x \in B}\{\sup \{|y|$ : $y \in G(x)\}\}<\infty$ ). $G$ is called upper semicontinuous (u. s. c.) on $X$ if for each $x_{*} \in X$ the set $G\left(x_{*}\right)$ is a nonempty, closed subset of $X$, and if for each open set $B$ of $X$ containing $G\left(x_{*}\right)$, there exists an open neighbourhood $V$ of $x_{*}$ such that $G(V) \subseteq B$. $G$ is said to be completely continuous if $G(B)$ is relatively compact for every bounded subset $B \subseteq X$. If the multivalued map $G$ is completely continuous with nonempty compact values, then $G$ is u. s. c. if and only if $G$ has a closed graph (i.e. $x_{n} \rightarrow$ $x_{*}, y_{n} \rightarrow y_{*}, y_{n} \in G\left(x_{n}\right)$ imply $\left.y_{*} \in G\left(x_{*}\right)\right)$. $G$ has a fixed point if there is $x \in X$ such that $x \in G(x)$.

In what follows, $C C(X)$ denotes the set of all nonempty compact and convex subsets of $X$.

A multivalued map $G: \mathcal{J} \rightarrow C C(X)$ is said to be measurable if for each $w \in X$ the function $Y: \mathcal{J} \rightarrow \mathbb{R}$ defined by

$$
Y(x, y)=d(w, G(x, y))=\inf \{|w-v|: v \in G(x, y)\}
$$

is measurable.

Definition 2.1. A multivalued map $F: \mathcal{J} \times \mathbb{R} \rightarrow 2^{\mathbb{R}}$ is said to be an $L^{1}$-Carathéodory map if

(i) $(x, y) \mapsto F(x, y, u)$ is measurable for each $u \in \mathbb{R}$;

(ii) $u \mapsto F(x, y, u)$ is upper semicontinuous for almost all $(x, y) \in \mathcal{J}$;

(iii) For each $k>0$, there exists $h_{k} \in L^{1}\left(\mathcal{J}, \mathbb{R}_{+}\right)$such that

$$
\|F(x, y, u)\|=\sup \{|v|: v \in F(x, y, u)\} \leq h_{k}(x, y)
$$

for all $|u| \leq k$ and for almost all $(x, y) \in \mathcal{J}$. 
An upper semi-continuous map $G: X \rightarrow 2^{X}$ is said to be condensing if for any subset $B \subseteq X$ with $\alpha(B) \neq 0$, we have $\alpha(G(B))<\alpha(B)$, where $\alpha$ denotes the Kuratowski measure of noncompactness. For properties of the Kuratowski measure, we refer to Banas and Goebel [3].

We remark that a completely continuous multivalued map is the easiest example of a condensing map. For more details on multivalued maps see the books by Deimling [9] and $\mathrm{Hu}$ and Papageorgiou [11].

Definition 2.2. By a solution of (1.1), (1.2) we mean a function $u(\cdot, \cdot) \in C(\mathcal{J}, \mathbb{R})$ such that there exists $v \in L^{1}(\mathcal{J}, \mathbb{R})$ for which we have

$$
u(x, y)=f(x)+g(y)-f(0)+\int_{0}^{x} \int_{0}^{y} v(t, s) d t d s \text { for each }(x, y) \in \mathcal{J}
$$

and with $v(t, s) \in F(t, s, u(t, s))$ a. e. on $\mathcal{J}$.

Now, we introduce the concept of lower and upper solutions to (1.1), (1.2). It will be the basic tool in the approach that follows.

Definition 2.3. A function $\underline{u}(\cdot, \cdot) \in C(\mathcal{J}, \mathbb{R})$ is said to be a lower solution of (1.1), (1.2) if there exists $v_{1} \in L^{1}(\mathcal{J}, \mathbb{R})$ such that $v_{1}(t, s) \in F(t, s, \underline{u}(t, s))$ a. e. on $\mathcal{J}$, and for which we have

$$
\underline{u}(x, y) \leq f(x)+g(y)-f(0)+\int_{0}^{x} \int_{0}^{y} v_{1}(t, s) d t d s \text { for each }(x, y) \in \mathcal{J} .
$$

A function $\bar{u}(\cdot, \cdot) \in C(\mathcal{J}, \mathbb{R})$ is said to be an upper solution of (1.1), (1.2) if there exists $v_{2} \in L^{1}(\mathcal{J}, \mathbb{R})$ such that $v_{2}(t, s) \in F(t, s, \bar{u}(t, s))$ a. e. on $\mathcal{J}$, and for which we have

$$
\bar{u}(x, y) \geq f(x)+g(y)-f(0)+\int_{0}^{x} \int_{0}^{y} v_{2}(t, s) d t d s \text { for each }(x, y) \in \mathcal{J} .
$$

For the multivalued map $F$ and for each $u \in C(\mathcal{J}, \mathbb{R})$ we define $S_{F, u}^{1}$ by

$$
S_{F, u}^{1}=\left\{v \in L^{1}(\mathcal{J}, \mathbb{R}): v(t, s) \in F(t, s, u(t, s)) \text { for a. e. }(t, s) \in \mathcal{J}\right\} .
$$

Our considerations are based on the following lemmas.

Lemma 2.4 ([16]). Let $F$ be a multivalued map satisfying (H1) and let $\Gamma$ be a linear continuous mapping from $L^{1}(\mathcal{J}, \mathbb{R})$ to $C(\mathcal{J}, \mathbb{R})$, then the operator

$$
\Gamma \circ S_{F}: C(\mathcal{J}, \mathbb{R}) \rightarrow C C(C(\mathcal{J}, \mathbb{R})), \quad u \longmapsto\left(\Gamma \circ S_{F}\right)(u):=\Gamma\left(S_{F, u}\right)
$$

is a closed graph operator in $C(\mathcal{J}, \mathbb{R}) \times C(\mathcal{J}, \mathbb{R})$.

Lemma 2.5 ([17]). Let $X$ be a Banach space and $N: X \rightarrow C C(X)$ be an u. s. . and condensing map. If the set

$$
\Omega:=\{u \in X: \lambda u \in N(u) \text { for some } \lambda>1\}
$$

is bounded, then $N$ has a fixed point. 
We will need the following hypotheses:

(H1) $F: \mathcal{J} \times \mathbb{R} \rightarrow C C(\mathbb{R})$ is an $L^{1}$ - Carathéodory multivalued map.

(H2) there exist $\underline{u}$ and $\bar{u}$ in $C(\mathcal{J}, \mathbb{R})$ as lower and upper solutions, respectively, to the problem (1.1), (1.2) such that $\underline{u} \leq \bar{u}$.

(H3) The functions $f, g: J \rightarrow \mathbb{R}$ are continuous with $f(0)=g(0)$.

\section{Main Result}

Now, we are able to state and prove our main theorem.

Theorem 3.1. Assume that hypotheses (H1)-(H3) hold. Then problem (1.1), (1.2) has at least one solution on $\mathcal{J}$.

Proof. Let us transform problem (1.1), (1.2) into a fixed point problem. Consider the following modified problem

$$
\begin{gathered}
\frac{\partial^{2} u(x, y)}{\partial x \partial y} \in F(x, y,(\tau u)(x, y)), \quad(x, y) \in \mathcal{J} \\
u(x, 0)=f(x), \quad u(0, y)=g(y)
\end{gathered}
$$

where $\tau: C(\mathcal{J}, \mathbb{R}) \rightarrow C(\mathcal{J}, \mathbb{R})$ is the truncation operator defined by

$$
(\tau u)(x, y)= \begin{cases}\underline{u}(x, y) & \text { if } u(x, y)<\underline{u}(x, y) ; \\ u(x, y) & \text { if } \underline{u}(x, y) \leq u \leq \bar{u}(x, y) ; \\ \bar{u}(x, y)) & \text { if } \bar{u}(x, y)<u(x, y) .\end{cases}
$$

Set

$$
C_{0}(\mathcal{J}, \mathbb{R}):=\{u \in C(\mathcal{J}, \mathbb{R}): u(x, 0)=f(x), u(0, y)=g(y)\} .
$$

A solution to (3.1), (3.2) is a fixed point of the operator $N: C_{0}(\mathcal{J}, \mathbb{R}) \rightarrow 2^{C_{0}(\mathcal{J}, \mathbb{R})}$ defined by

$$
\begin{array}{r}
N(u):=\left\{h \in C_{0}(\mathcal{J}, \mathbb{R}): h(x, y)=f(x)+g(y)-f(0)+\int_{0}^{x} \int_{0}^{y} v(t, s) d t d s,\right. \\
\left.v \in \tilde{S}_{F, \tau u}^{1}\right\}
\end{array}
$$

where

$$
\begin{gathered}
\tilde{S}_{F, \tau u}^{1}=\left\{v \in S_{F, \tau u}^{1}: v(t, s) \geq v_{1}(t, s) \text { a. e. on } A_{1} \text { and } v(t, s) \leq v_{2}(t, s) \text { a. e. on } A_{2}\right\}, \\
S_{F, \tau u}^{1}=\left\{v \in L^{1}(\mathcal{J}, \mathbb{R}): v(t, s) \in F(t, s,(\tau u)(t, s)) \text { for a. e. }(t, s) \in \mathcal{J}\right\} \\
A_{1}=\{(t, s) \in \mathcal{J}: u(t, s)<\underline{u}(t, s) \leq \bar{u}(t, s)\} \\
A_{2}=\{(t, s) \in \mathcal{J}: \underline{u}(t, s) \leq \bar{u}(t, s)<u(t, s)\}
\end{gathered}
$$

Remark 3.2. (i) For every $u \in C(\mathcal{J}, \mathbb{R})$, the set $S_{F, u}^{1}$ is nonempty (see Lasota and Opial [16]). 
(ii) For every $u \in C(\mathcal{J}, \mathbb{R})$, the set $\tilde{S}_{F, \tau u}^{1}$ is nonempty. Indeed, by (i) there exists $v \in S_{F, u}^{1}$. Set $w=v_{1} \chi_{A_{1}}+v_{2} \chi_{A_{2}}+v \chi_{A_{3}}$, where

$$
A_{3}=\{(t, s) \in \mathcal{J}: \underline{u}(t, s) \leq u(t, s) \leq \bar{u}(t, s)\} .
$$

Then, by decomposability, $w \in \tilde{S}_{F, \tau u}^{1}$.

We shall show that $N$ is a completely continuous multivalued map, u. s. c. with convex closed values. The proof will be divided into several steps.

Remark 3.3. It is clear that the fixed points of $N$ are solutions to (3.1), (3.2).

STEP 1: $N(u)$ is convex for each $u \in C(\mathcal{J}, \mathbb{R})$.

Indeed, if $h_{1}, h_{2}$ belong to $N(u)$, then there exist $v^{1}, v^{2} \in \tilde{S}_{F, \tau u}$ such that for each $(x, y) \in \mathcal{J}$ we have

$$
h_{i}(x, y)=f(x)+g(y)-f(0)+\int_{0}^{x} \int_{0}^{y} v^{i}(t, s) d t d s, \quad i=1,2 .
$$

Let $0 \leq \alpha \leq 1$. Then for each $(x, y) \in \mathcal{J}$ we have

$$
\begin{aligned}
\left(\alpha h_{1}+(1-\alpha) h_{2}\right)(x, y)=f(x)+g(y)- & f(0) \\
& +\int_{0}^{x} \int_{0}^{y}\left[\alpha v^{1}(t, s)+(1-\alpha) v^{2}(t, s)\right] d s .
\end{aligned}
$$

Since $\tilde{S}_{F, \tau u}$ is convex (because $F$ has convex values), we have $\alpha h_{1}+(1-\alpha) h_{2} \in N(u)$.

STEP 2: $N$ is bounded on bounded sets of $C(\mathcal{T}, \mathbb{R})$.

Indeed, it is enough to show that there exists a positive constant $c$ such that for each $h \in N(u), u \in B_{r}=\left\{u \in C(\mathcal{J}, \mathbb{R}):\|u\|_{\infty} \leq r\right\}$ one has $\|h\|_{\infty} \leq c$.

If $h \in N(u)$, then there exists $v \in \tilde{S}_{F, \tau u}$ such that for each $(x, y) \in \mathcal{J}$ we have

$$
h(x, y)=f(x)+g(y)-f(0)+\int_{0}^{x} \int_{0}^{y} v(t, s) d t d s .
$$

By (H1) we have for each $(x, y) \in \mathcal{J}$ that

$$
|h(x, y)| \leq|f(x)|+|g(y)|+|f(0)|+\int_{0}^{x} \int_{0}^{y} h_{r}(t, s) d t d s .
$$

Then

$$
\|h\|_{\infty} \leq\|f\|_{\infty}+\|g\|_{\infty}+|f(0)|+\int_{0}^{T} \int_{0}^{T} h_{r}(t, s) d t d s=c .
$$

StEP 3: $N$ sends bounded sets of $C(\mathcal{J}, \mathbb{R})$ into equicontinuous sets.

Let $\left(x_{1}, y_{1}\right),\left(x_{2}, y_{2}\right) \in \mathcal{J}, x_{1}<x_{2}, y_{1}<y_{2}$ and $B_{r}$ be a bounded set of $C(\mathcal{J}, \mathbb{R})$. For each $u \in B_{r}$ and $h \in N(u)$, there exists $v \in \tilde{S}_{F, \tau u}$ such that

$$
h(x, y)=f(x)+g(y)-f(0)+\int_{0}^{x} \int_{0}^{y} v(t, s) d t d s .
$$


Thus we obtain

$$
\begin{aligned}
\left|h\left(x_{2}, y_{2}\right)-h\left(x_{1}, y_{1}\right)\right| & \leq\left|f\left(x_{2}\right)-f\left(x_{1}\right)\right|+\left|g\left(y_{2}\right)-g\left(y_{1}\right)\right|+\int_{x_{1}}^{x_{2}} \int_{y_{1}}^{y_{2}}|v(t, s)| d t d s \\
& \leq\left|f\left(x_{2}\right)-f\left(x_{1}\right)\right|+\left|g\left(y_{2}\right)-g\left(y_{1}\right)\right|+\int_{x_{1}}^{x_{2}} \int_{y_{1}}^{y_{2}} h_{r}(t, s) d t d s .
\end{aligned}
$$

As $\left(x_{2}, y_{2}\right) \rightarrow\left(x_{1}, y_{1}\right)$, the right-hand side of the above inequality tends to zero.

Taking into account Step 2, Step 3, and the Arzelà-Ascoli theorem, we conclude that $N$ is completely continuous and therefore a condensing multivalued map.

STEP 4: $N$ has closed graph.

Let $u_{n} \rightarrow u_{*}, h_{n} \in N\left(u_{n}\right)$, and $h_{n} \rightarrow h_{*}$. We shall prove that $h_{*} \in N\left(u_{*}\right)$. The inclusion $h_{n} \in N\left(u_{n}\right)$ means that there exists $v_{n} \in \tilde{S}_{F, \tau u_{n}}$ such that

$$
h_{n}(x, y)=f(x)+g(y)-f(0)+\int_{0}^{x} \int_{0}^{y} v_{n}(t, s) d t d s,(x, y) \in \mathcal{J} .
$$

We must prove that there exists $g_{*} \in \tilde{S}_{F, \tau u_{*}}$ such that

$$
h_{*}(x, y)=f(x)+g(y)-f(0)+\int_{0}^{x} \int_{0}^{y} v_{*}(t, s) d t d s, \quad(x, y) \in \mathcal{J} .
$$

Now, we consider the linear continuous operator $\Gamma: L^{1}(\mathcal{J}, \mathbb{R}) \rightarrow C(\mathcal{J}, \mathbb{R})$,

$$
v \longmapsto \Gamma(v)(x, y)=\int_{0}^{x} \int_{0}^{y} v(t, s) d t d s,(x, y) \in \mathcal{J} .
$$

From Lemma 2.4, it follows that $\Gamma \circ \tilde{S}_{F}$ is a closed graph operator.

Clearly we have

$$
\left\|\left(h_{n}(x, y)-f(x)-g(y)+f(0)\right)-\left(h_{*}(x, y)-f(x)-g(y)+f(0)\right)\right\|_{\infty} \rightarrow 0
$$

as $n \rightarrow \infty$. Moreover, from the definition of $\Gamma$, we have

$$
\left(h_{n}(x, y)-f(x)-g(y)+f(0)\right) \in \Gamma\left(\tilde{S}_{F, \tau u_{n}}\right) .
$$

Since $u_{n} \rightarrow u_{*}$, it follows from Lemma 2.4 that, for some $v_{*} \in \tilde{S}_{F, \tau u_{*}}$,

$$
h_{*}(x, y)-f(x)-g(y)+f(0)=\int_{0}^{x} \int_{0}^{y} v_{*}(t, s) d t d s, \quad(x, y) \in \mathcal{J} .
$$

STEP 5: The set

$$
\Omega:=\{u \in C(\mathcal{J}, \mathbb{R}): \lambda u \in N(u) \text { for some } \lambda>1\}
$$

is bounded.

Let $u \in \Omega$. Then $\lambda u \in N(u)$ for some $\lambda>1$. Thus there exists $v \in \tilde{S}_{F, \tau u}$ such that

$$
u(x, y)=\lambda^{-1} f(x)+\lambda^{-1} g(y)-\lambda^{-1} f(0)+\lambda^{-1} \int_{0}^{x} \int_{0}^{y} v(t, s) d t d s, \quad(x, y) \in \mathcal{J} .
$$


This implies that for each $(x, y) \in \mathcal{J}$ we have

$$
|u(x, y)| \leq|f(x)|+|g(y)|+|f(0)|+\int_{0}^{x} \int_{0}^{y}|v(t, s)| d t d s .
$$

The definition of $\tau$ implies that there exists $\phi \in L^{1}\left(\mathcal{J}, \mathbb{R}^{+}\right)$such that $\|F(x, y, \tau u)\|=$ $\sup \{|v|: v \in F(x, y, \tau u)\} \leq \phi(x, y)$ for each $u \in C(\mathcal{J}, \mathbb{R})$. Thus we obtain

$$
\|u\|_{\infty} \leq\|f\|_{\infty}+\|g\|_{\infty}+|f(0)|+\int_{0}^{T} \int_{0}^{T} \phi(t, s) d t d s=K .
$$

This shows that $\Omega$ is bounded. Set $X:=C(\mathcal{J}, \mathbb{R})$. As a consequence of Lemma 2.5 we deduce that $N$ has a fixed point which is a solution of (3.1), (3.2) on $\mathcal{J}$.

STEP 6: The solution $u$ of (3.1), (3.2) satisfies the relations

$$
\underline{u}(x, y) \leq u(x, y) \leq \bar{u}(x, y) \text { for all }(x, y) \in \mathcal{J} .
$$

Let $u$ be a solution to (3.1), (3.2). We prove that $\underline{u}(x, y) \leq u(x, y)$ for all $(x, y) \in \mathcal{J}$.

Let us suppose the contrary. Then there exist $x_{1}, x_{2}, y_{1}, y_{2} \in J, x_{1}<x_{2}, y_{1}<y_{2}$ such that $\underline{u}\left(x_{1}, y_{1}\right)=u\left(x_{1}, y_{1}\right)$ and $\underline{u}(x, y)>u(x, y)$ for all $(x, y) \in\left(x_{1}, x_{2}\right) \times\left(y_{1}, y_{2}\right)$. In view of the definition of $\tau$ there exists $v(\cdot, \cdot) \in F(\cdot, \cdot, \underline{u}(\cdot, \cdot))$ a. e. on $\mathcal{J}$ with $v(x, y) \geq$ $v_{1}(x, y)$ a. e. on $\left(x_{1}, x_{2}\right) \times\left(y_{1}, y_{2}\right)$ such that

$$
u(x, y)=f(x)+g(y)-f(0)+\int_{0}^{x} \int_{0}^{y} v(t, s) d t d s, \quad(x, y) \in\left(x_{1}, x_{2}\right) \times\left(y_{1}, y_{2}\right) .
$$

Since $\underline{u}$ is a lower solution to (1.1), (1.2), then

$$
\underline{u}(x, y) \leq f(x)+g(y)-f(0)+\int_{0}^{x} \int_{0}^{y} v_{1}(t, s) d t d s \quad(x, y) \in\left(x_{1}, x_{2}\right) \times\left(y_{1}, y_{2}\right) .
$$

It follows from the facts $u\left(x_{1}, y_{1}\right)=\underline{u}\left(x_{1}, y_{1}\right), v(x, y) \geq v_{1}(x, y)$ that

$$
\underline{u}(x, y) \leq u(x, y) \text { for all }(x, y) \in\left(x_{1}, x_{2}\right) \times\left(y_{1}, y_{2}\right),
$$

which is a contradiction since $u(x, y)<\underline{u}(x, y)$ for all $(x, y) \in\left(x_{1}, x_{2}\right) \times\left(y_{1}, y_{2}\right)$. Consequently, $\underline{u}(x, y) \leq u(x, y)$ for all $(x, y) \in \mathcal{J}$.

Analogously, we can prove that $u(x, y) \leq \bar{u}(x, y)$ for all $(x, y) \in \mathcal{J}$. This shows that problem (3.1), (3.2) has a solution in the interval $[\underline{u}, \bar{u}]$. Since $\tau(u)=u$ for all $u \in[\underline{u}, \bar{u}]$, we see that $u$ is a solution to $(1.1),(1.2)$.

\section{REFERENCES}

[1] Agarwal, R. P.: The method of upper, lower solutions and monotone iterative scheme for high order hyperbolic partial differential equations, J. Austral. Math. Soc. (Series A), 47 (1989), 153170.

[2] Agarwal, R. P. and Sheng, Q., Periodic solutions of higher order hyperbolic partial differential equations, PanAmer. Math. J., 2 (1992), 1-22.

[3] Banas, J. And Goebel, K.: Measures of Noncompactness in Banach Spaces, Marcel Dekker, New York, 1980. 
[4] Benchohra, M. and Ntouyas, S. K.: An existence theorem for an hyperbolic differential inclusion in Banach spaces, Discuss. Math., Differ. Incl., 22 (2002), 5-16.

[5] Blakley, R. D. and Pandit, S. G.: On a sharp linear comparison result and an application to nonlinear Cauchy problem, Dynam. Systems Appl., 3 (1994), 135-140.

[6] Carl, S. and Heik kilae, S.: Nonlinear Differential Equations in Ordered Spaces, Chapman \& Hall, 2000.

[7] De Blasi, F. and Myjak, J.: On the structure of the set of solutions of the Darboux problem for hyperbolic equations, Proc. Edinburgh Math. Soc. 29 (1986), 7-14.

[8] De Blasi, F. and MyjaK, J.: On the set of solutions of a differential inclusion, Bull. Inst. Math., Acad. Sin., 14 (1986), 271-275.

[9] Deimling, K.: Multivalued Differential Equations, Walter de Gruyter, Berlin - New York, 1992.

[10] Heik kilae, S. and Lakshmikantham, V.: Monotone Iterative Techniques for Discontinuous Nonlinear Differential Equations, Pure and Applied Mathematics, Marcel Dekker, New York, 1994.

[11] Hu, Sh. And Papageorgiou, N.: Handbook of Multivalued Analysis, Vol. I, Kluwer AP, Dordrecht, Boston, and London, 1997.

[12] Kamont, Z.: Hyperbolic Functional Differential Inequalities and Applications, Mathematics and Applications, 486, Dordrecht, 1999.

[13] Kuвiaczyк, I.: Knesner's theorem for hyperbolic equations, Funct. Approx. Comment. Math., 14 (1984), 183-196.

[14] Lakshmikantham, V. and Pandit, S. G.: The method of upper, lower solutions and hyperbolic partial differential equations, J. Math. Anal. Appl., 105 (1985), 466-477.

[15] Ladde, G. S., Lakshmikantham, V., and Vatsala, A. S.: Monotone Iterative Techniques for Nonlinear Differential Equations, Pitman, Boston, 1995.

[16] Lasota, A. and Opial, Z.: An application of the Kakutani - Ky Fan theorem in the theory of ordinary differential equations, Bull. Acad. Polon. Sci. Ser. Sci. Math. Astron. Phys., 13 (1965), 781-786.

[17] Martelli, M.: A Rothe's type theorem for noncompact acyclic-valued map, Boll. Un. Math. Ital., 11 (1975), 70-76.

[18] Pandit, S. G.: Monotone methods for systems of nonlinear hyperbolic problems in two independent variables, Nonlinear Anal., 30 (1997), 2735-272.

[19] Papageorgiou, N. S.: Existence of solutions for hyperbolic differential inclusions in Banach spaces, Arch. Math. (Brno), 28 (1992), 205-213.

\section{Authors' Addresses}

\section{Benchohra:}

Department of Mathematics, University of Sidi Bel Abbes, BP 89, 22000 Sidi Bel Abbes, Algeria

E-mail address: benchohra@yahoo.com

\section{S. K. Ntouyas:}

Department of Mathematics, University of Ioannina, 45110 Ioannina, Greece

E-mail address: sntouyas@cc.uoi.gr 